\title{
Simulación del proceso de biodegradación de aguas residuales de la industria de carne mediante una red neuronal artificial perceptrón multicapa
}

\author{
Simulation of biodegradation process of wastewater from meat industry \\ by means of a multilayer perceptron artificial neural network
}

José C. Curvelo Santana ${ }^{1} \quad$ Sidnei A. de Araújo ${ }^{1} \quad$ Joana P. M. Biazus ${ }^{2} \quad$ Roberto R. de Souza ${ }^{2}$

Recibido 30 de septiembre de 2013, aceptado 29 de julio de 2014

Received: September 30, 2013 Accepted: July 29, 2014

\begin{abstract}
RESUMEN
En este trabajo se propone utilizar una Red Neuronal Artificial (RNA) Perceptrón Multicapa (PMC) para simular la variación de la concentración de proteína de acuerdo con el tiempo y también para determinar la hora final del procedimiento, además de los parámetros óptimos del proceso de biodegradación de las proteínas de un efluente de matadero. Para eso, han sido utilizadas las papaínas, presentes en el látex de la papaya (Carica papaya) con el objetivo de disminuir la concentración de proteínas de un efluente de matadero a pH (5 y 7) con una temperatura de $\left(25\right.$ y $\left.30^{\circ} \mathrm{C}\right)$ controlada. Los resultados mostraron que las papaínas redujeron de $82 \%$ a $91 \%$ la concentración de proteína en 30 y 40 h de proceso. Las simulaciones con la RNA apuntaron que las condiciones perfectas fueron obtenidas a $\mathrm{pH} 5$, con $30^{\circ} \mathrm{C}$ y en $35 \mathrm{~h}$, en el cual se ha alcanzado una reducción de $91 \%$ de la concentración de proteínas.
\end{abstract}

Palabras clave: Redes neuronales, perceptrón multicapa, simulación, tratamiento de efluentes, proteínas.

\section{ABSTRACT}

In this paper, the use of a multilayer perceptron (MLP) artificial neural network (ANN) is proposed to simulate the variation of protein concentration according to the time and also to determine the end and optimal conditions of the biodegradation process of wastewater from meat industry. To reduce the protein concentration, papains from Carica papaya latex have been used at controlled condition of $p H$ ( 5 and 7$)$ and temperature $\left(25\right.$ and $\left.30^{\circ} \mathrm{C}\right)$. Results showed that a reduction of 82 to $91 \%$ of protein concentration by the action of papains for 30 to 40 h of process time. Simulations showed that the best condition of the process occurred at $\mathrm{pH} 5,30^{\circ} \mathrm{C}$ and $35 \mathrm{~h}$, in which a maximum biodegradation of $91 \%$ was obtained.

Keywords: Neural network, multilayer perceptron, simulation, effluent treatment, proteins.

\section{INTRODUCCIÓN}

Actualmente, existe una preocupación creciente acerca de la preservación del medio ambiente. Así, hay varias investigaciones que tratan del tema de la reducción de los residuos industriales, visando la preservación del agua y del suelo en todo el mundo
[1-3]. De acuerdo con [4-5] una alternativa para la valorización de residuos es el desarrollo de nuevos productos, dándoles un destino más noble y con un gran valor comercial. Cuando el residuo o efluente no pueden ser reutilizados, se hace necesario tratarlos, como en los casos citados por [6] que produjeron un biodiesel por medio del aceite de cocina utilizado en

\footnotetext{
1 Programa de Pos-Graduação em Engenharia de Produção. Universidade Nove de Julho (UNINOVE). Av. Francisco Matarazzo, 612, 05001-100. São Paulo, SP, Brasil. E-mail: jccurvelo@uninove.br; saraujo@uninove.br

2 Departamento de Engenharia Química. Universidade Federal de Sergipe (UFS). Av. Marechal Rondon, s/n, Jardim Rosa Elze, 49100-000. São Cristóvão, SE, Brasil. E-mail: biazzusjp@gmail.com; rrsouza@ufs.br
} 
las residencias, por [7] que reutilizaron las cáscaras de naranja para la obtención de aceites esenciales, para agregar valor a estos residuos y por [1] que desarrollaron un estudio sobre la remediación de suelos accidentalmente contaminados con ácido sulfúrico mediante métodos geofísicos.

El efluente de la industria de procesamiento de carne tiene una demanda biológica de oxígeno (DBO) de 1.400 a $11.000 \mathrm{mg} / \mathrm{L}$ y es de 70 a 200 veces más contaminante que las aguas residuales. Los mataderos generan efluentes líquidos compuestos de grasas, proteínas y restos de animales. Además, generan desechos sólidos, tales como: huesos, entrañas, patas, cabezas, piel y otros restos de carcasas. En este contexto, se hace necesario buscar alternativas de aprovechamiento y reutilización de los residuos obtenidos en los mataderos.

En [4-5, 8-9] se muestra cómo reutilizar las patas de pollo para obtener gelatinas. Sin embargo, en Brasil, los efluentes cárnicos casi no son tratados, sino descartados in natura en los cuerpos de agua. En otros campos de investigación se observó los progresos realizados para reducir, al mínimo, los efectos de las descargas de efluentes, como los casos citados por [2, 10-14] que utilizaron varias técnicas para el tratamiento y reutilización de las aguas residuales, en la irrigación de plantas y recuperación de suelos; o por [3] que realizaron la biosorción de metales en cepas de levaduras de cerveza. O incluso en el caso de [15] que aplicaron las cepas de los microorganismos para el tratamiento de aguas residuales de petróleo.

Las Redes Neuronales Artificiales (RNA) han contribuido con diversos campos de la investigación, en lo que se refiere a la solución de problemas complejos. En la ingeniería mecánica, como se ha señalado en el trabajo desarrollado por [16] ha sido presentada una metodología para análisis de la rugosidad basada en las características superficiales de las imágenes, obtenidas en microscopios ópticos y electrónicos, en el cual los valores extraídos de los patrones de rugosidad primaria se incorporan después a una RNA de Perceptrón Multicapa (PMC). En la ingeniería de organización, como el trabajo llevado a cabo por [17] se detalla el mecanismo automatizado por una RNA, en el que se determina el estado de la iluminación, temperatura, humedad y otros valores de entorno y en cómo se actúa sobre los patrones de comportamiento del usuario. En la ciencia de alimentos, los trabajos [18] y [9], que han presentado una RNA basada en los algoritmos de Kohonen para el agrupamiento de alimentos por sus calidades sensoriales.

En la literatura reciente, existen varios trabajos que proponen el uso de redes neuronales artificiales en la optimización de los parámetros en los procesos de biodegradación de aguas residuales, especialmente en los sectores textil, metalúrgico, químico y farmacéutico. Entre ellas podemos mencionar [19-25].

En este trabajo se utilizó una RNA del tipo PMC para simular la concentración de proteína, de acuerdo con el tiempo y también para determinar la hora final del procedimiento, además de los parámetros óptimos del proceso de biodegradación. Sin embargo, en la revisión de la literatura no se encontraron estudios que traten del proceso de tratamiento de aguas residuales de la industria de carne, tampoco sobre los mismos parámetros investigados en este trabajo, lo que nos impidió de hacer una comparación de los resultados obtenidos.

\section{MATERIALES Y MÉTODOS}

\section{Enzimas y biorreactor}

De las enzimas se extrajeron $10 \mathrm{~g}$ de látex de papaya verde (Carica papaya) mezclada con $100 \mathrm{~mL}$ de una solución a 3\% de $\mathrm{NaCl}$ y enseguida se centrifugó a $3000 \mathrm{rpm}$, durante $15 \mathrm{~min}$. El sobrenadante se consideró como enzimas papaínas y se almacenó a $5{ }^{\circ} \mathrm{C}$ [26]. El birreactor operó hasta la degradación completa de las proteínas o la inactivación de las enzimas. El efluente fue esterilizado en un autoclave y diluido a $555 \mathrm{mg} / \mathrm{L}$ de concentración de proteína usando tampón de fosfato a $0,2 \mathrm{M}$ (en el $\mathrm{pH} 5 \mathrm{o}$ 7). Esto fue utilizado como una concentración inicial estándar $\left(\mathrm{C}_{0}\right)$, en el cual se colocó en matraces de Erlenmeyer de $250 \mathrm{~mL}$, y que se agitó constantemente. Estos frascos fueron colocados en baños termales (a 25 o $30^{\circ} \mathrm{C}$ ) y tras la temperatura se estabilizaran, se añadió $5 \mathrm{ml}$ de solución de la enzima de papaína, considerado como el comienzo del proceso de biodegradación [26-27].

La concentración de proteína se determina a partir de la reacción de la biodegradación, conforme el método de Bradford [28] y es expresado en forma adimensional $\left(C_{a d}\right)$, como se presenta en 
ecuación (1) para comprobar que la actividad de la enzima se mantuvo. Para verificar la eficiencia del proceso de biodegradación se utiliza la ecuación (2), como se muestra a continuación. Donde, $C_{0}$ es la concentración inicial y $C_{i}$ es la concentración medida esporádicamente.

$$
\begin{gathered}
C_{a d}=\frac{C_{i}}{C_{0}} \\
\text { Bio degradación }=100 *\left(1 \frac{C_{i}}{C_{0}}\right)
\end{gathered}
$$

\section{Configuraciones de la RNA}

El PMC es un modelo de RNA caracterizado por tener, además de la entrada y salida, una o más capas ocultas. Para entrenamiento, este modelo de red neuronal, en general, utiliza un algoritmo supervisado, conocido como backpropagation. Este algoritmo se basa en el aprendizaje de la corrección de errores y es compuesto de dos fases: la propagación y la retropropagación. En la primera fase, cuando se presenta un patrón para la red, por medio de su capa de entrada, la señal de activación se propaga capa por capa, hasta que la respuesta se produzca por la capa de salida. En la segunda etapa, se compara la salida obtenida con la salida deseada para el patrón de entrada presentado, y se produce una señal de error, que se propaga. Enseguida la capa de salida y de entrada y los pesos sinápticos se ajustan de modo que la respuesta de la red se acerca de la respuesta deseada.

Para lograr un alto grado de aprendizaje en la red, es necesario que se haga un buen entrenamiento con varios patrones diferentes. Cuanto más grande el número de ejemplos que se presentan en la red de formación, mejor el rendimiento. Un entrenamiento con pocos ejemplos puede ser más rápido, pero puede causar inestabilidad en el funcionamiento de la red [29].

Para simular la variación de la concentración de proteínas de acuerdo con el tiempo del processo se construyó una RNA, modelo PMC, usando el lenguaje de programación $\mathrm{C} / \mathrm{C}++$, con 3 neuronas en la capa de entrada, 15 en la capa oculta y 1 en la capa de salida.

El número de neuronas en las capas de entrada y salida están de acuerdo con los datos utilizados en el entrenamiento, presentados en la Tabla 1. Para obtener el número de neuronas de la capa oculta, varios experimentos se llevaron a cabo mediante la variación de este parámetro, y luego fue elegida la arquitectura de red que dio lugar a un error cuadrático medio (ECM) en la fase de aprendizaje. Otros parámetros utilizados en el entrenamiento de la red neuronal propuesta fueron: i) función de transferencia: Logística, ii) tasa de aprendizaje de 0,1 , iii) tasa de momento: 0,9 , iv) criterio de parada: ECM de $10^{-5}[16,29]$.

Para entrenamiento, la red neuronal incluye las 66 muestras descritas en la Tabla 1. Para usar la red para las simulaciones fueron utilizados diferentes tiempos a partir de los que se consideran en los ensayos. Los atributos de entrada de la RNA fueron: temperatura, $\mathrm{pH}$ y el tiempo. El atributo de salida es la concentración de proteína, conforme se ilustra en la Figura 1.

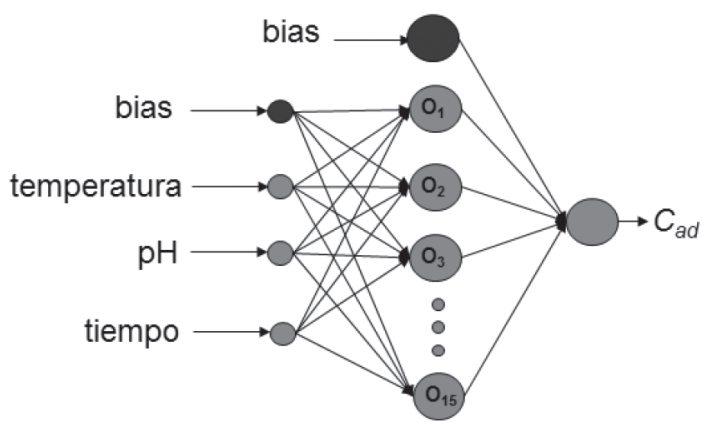

Figura 1. Arquitectura de la RNA propuesta.

\section{RESULTADOS Y DISCUSIÓN}

En las Figuras 2 y 3 se muestran las curvas de biodegradación obtenidas, tras medir a variación de la concentración de proteína de acuerdo con el tiempo del proceso, a los 25 y $30^{\circ} \mathrm{C}$, respectivamente, sobre los $\mathrm{pH} 5$ y 7 . Se observa en todas las figuras, que inicialmente hay una gran reducción de la concentración de proteína y luego una estabilización, después de $30 \mathrm{~h}$ para los ensayos a $30{ }^{\circ} \mathrm{C}$ y $40 \mathrm{~h}$ para los ensayos a $25^{\circ} \mathrm{C}$.

De acuerdo con Cadogan y Hanks [27] en una reacción enzimática la enzima se inhibe cuando se alcanza una concentración de saturación de sus sitios activos. Se puede verificar que la concentración de proteína en el extremo del proceso de biodegradación es más inferior en los ensayos a $30{ }^{\circ} \mathrm{C}$ que en 
Tabla 1. Datos recogidos en los ensayos y utilizados en el entrenamiento de la RNA.

\begin{tabular}{|c|c|c|c|}
\hline \multicolumn{4}{|c|}{ Temperatura $25^{\circ} \mathrm{C}$} \\
\hline \multicolumn{2}{|c|}{$\mathrm{pH}=5$} & \multicolumn{2}{|c|}{$\mathrm{pH}=7$} \\
\hline tiempo & $\mathrm{C}_{\mathrm{ad}}$ & tiempo & $\mathbf{C}_{\mathrm{ad}}$ \\
\hline 0 & 1,000 & 0 & 1,000 \\
\hline 5 & 0,670 & 5 & 0,705 \\
\hline 10 & 0,430 & 10 & 0,479 \\
\hline 15 & 0,38 & 15 & 0,465 \\
\hline 20 & 0,393 & 20 & 0,488 \\
\hline 25 & 0,264 & 25 & 0,404 \\
\hline 30 & 0,251 & 30 & 0,362 \\
\hline 35 & 0,253 & 35 & 0,375 \\
\hline 40 & 0,229 & 40 & 0,335 \\
\hline 45 & 0,155 & 45 & 0,284 \\
\hline 50 & 0,249 & 50 & 0,426 \\
\hline 55 & 0,173 & 55 & 0,321 \\
\hline 60 & 0,183 & 60 & 0,302 \\
\hline 65 & 0,132 & 65 & 0,227 \\
\hline 70 & 0,189 & 70 & 0,270 \\
\hline 76,2 & 0,180 & 76,2 & 0,261 \\
\hline 78,5 & 0,162 & 78,5 & 0,233 \\
\hline \multicolumn{4}{|c|}{ Temperatura $30^{\circ} \mathrm{C}$} \\
\hline \multicolumn{2}{|c|}{$\mathrm{pH}=5$} & \multicolumn{2}{|c|}{$\mathrm{pH}=7$} \\
\hline tiempo & $\mathrm{C}_{\mathrm{ad}}$ & tiempo & $\mathrm{C}_{\mathrm{ad}}$ \\
\hline 0 & 1,000 & 0 & 1,000 \\
\hline 5 & 0,433 & 3,5 & 0,601 \\
\hline 10 & 0,323 & 7 & 0,492 \\
\hline 15 & 0,272 & 10,5 & 0,439 \\
\hline 20 & 0,156 & 14 & 0,341 \\
\hline 25 & 0,187 & 17,5 & 0,308 \\
\hline 30 & 0,151 & 21 & 0,298 \\
\hline 35 & 0,118 & 24,5 & 0,271 \\
\hline 40 & 0,078 & 28 & 0,235 \\
\hline 45 & 0,026 & 31,5 & 0,170 \\
\hline 50 & 0,108 & 35 & 0,150 \\
\hline 55 & 0,109 & 38,5 & 0,117 \\
\hline 60 & 0,112 & 42 & 0,119 \\
\hline 65 & 0,095 & 45,5 & 0,132 \\
\hline 70 & 0,093 & 48,68 & 0,106 \\
\hline 80,25 & 0,102 & 80,25 & 0,102 \\
\hline
\end{tabular}

$25^{\circ} \mathrm{C}$. Los ensayos con un $\mathrm{pH}$ de 5 presentaron una reducción mayor en la concentración de proteínas que los ensayos con un $\mathrm{pH}$ de 7.

Esto se observa en la Tabla 2, que muestra los porcentajes de biodegradación para cada ensayo. También se puede observar que los ensayos hechos a $30{ }^{\circ} \mathrm{C}$ presentaron los mejores resultados, ya que hay una biodegradación de aproximadamente $90 \%$.

Tabla 2. Resultados del porcentaje de biodegradación.

\begin{tabular}{|c|c|c|c|c|}
\hline pH & \multicolumn{4}{|c|}{ Temperatura } \\
\hline & \multicolumn{2}{|c|}{$\mathbf{2 5}^{\circ} \mathbf{C}$} & \multicolumn{2}{c|}{$\mathbf{3 0}^{\circ} \mathbf{C}$} \\
\hline & $\begin{array}{c}\text { \% } \\
\text { Biodegradación }\end{array}$ & \pm & $\begin{array}{c}\text { \% } \\
\text { Biodegradación }\end{array}$ & \pm \\
\hline 5 & 82,208 & 3,411 & 90,970 & 2,807 \\
\hline 7 & 73,104 & 3,722 & 88,162 & 1,084 \\
\hline
\end{tabular}

Con la finalidad de evaluar la eficacia y la precisión de la RNA desarrollada después de su entrenamiento, la red ha sido utilizada para reconstruir las curvas de degradación de la proteína, utilizando el $\mathrm{pH}$ y la temperatura mencionados en la Tabla 1, pero con tiempos distintos de aquellos considerados en cada ensayo.

Después, las curvas generadas por la RNA fueron incorporadas en los mismos gráficos que muestran las curvas de la degradación de la proteína, obtenidas a partir de los datos recogidos en los ensayos (Figuras 2 y 3), lo que permite comparar, en términos de ECM, los resultados obtenidos por la red con variaciones en la concentración de proteína, observada en los ensayos.

Como se puede verificar en las Figuras 2 y 3, la RNA simuló el comportamiento de las curvas reales, incluso teniendo en cuenta diferentes tiempos de aquellos recogidos en los ensayos. Para las cuatro curvas, los ECM obtenidos fueron, respectivamente, 0,$005 ; 0,004 ; 0,001$ y 0,008 , lo que muestra la buena correlación que ocurrió con los datos de los ensayos a $30{ }^{\circ} \mathrm{C}$, con $\mathrm{pH} 5$.

Es importante destacar que las mayores diferencias que se muestran en las Figuras 2a, 2b y 3 b entre 40 h y $50 \mathrm{~h}$, puede indicar un problema en el proceso de recopilación de los datos. 

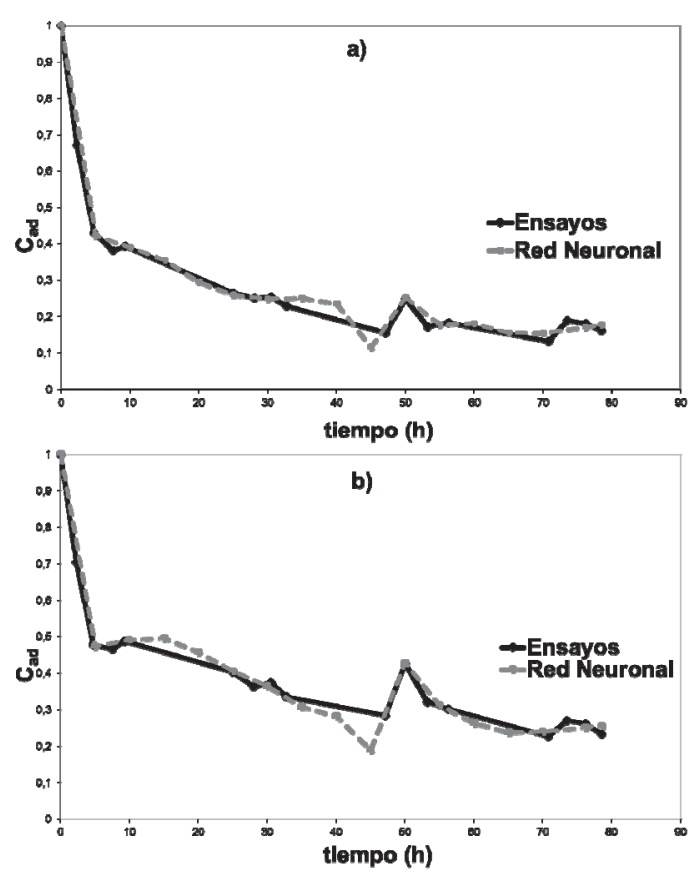

Figura 2. Curvas reales y simuladas de la biodegradación de proteínas por las papaínas a $25^{\circ} \mathrm{C} \mathrm{y} \mathrm{a)} \mathrm{pH} 5$, b) $\mathrm{pH} 7$.
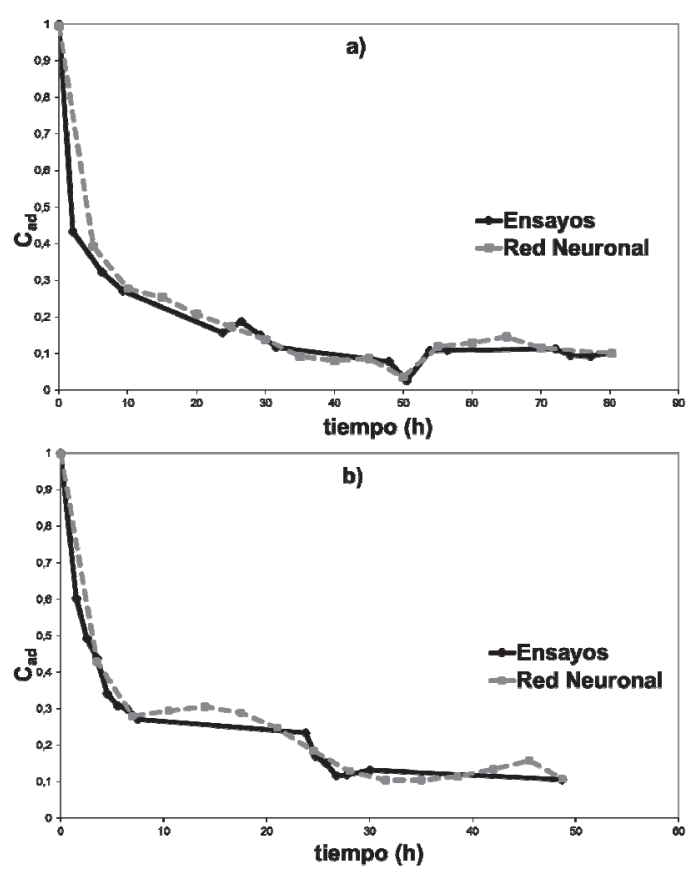

Figura 3. Curvas reales y simuladas de la biodegradación de proteínas por las papaínas a $30{ }^{\circ} \mathrm{C}$ y a) $\mathrm{pH} 5$, b) $\mathrm{pH} 7$.
Por fin, haciendo una simulación en la RNA con un conjunto determinado de temperatura y $\mathrm{pH}$, durante un período de tiempo más largo que el utilizado en los ensayos, se puede observar (Figura 4) que, tras algún tiempo, la respuesta de la RNA fue constante, lo que permite determinar el procedimiento final y las mejores condiciones de proceso de biodegradación. Importante resaltar que los pesos utilizados por la RNA en la simulación presentada en la Figura 4, son diferentes de los pesos usados para generar la curva mostrada en la Figura 3a.

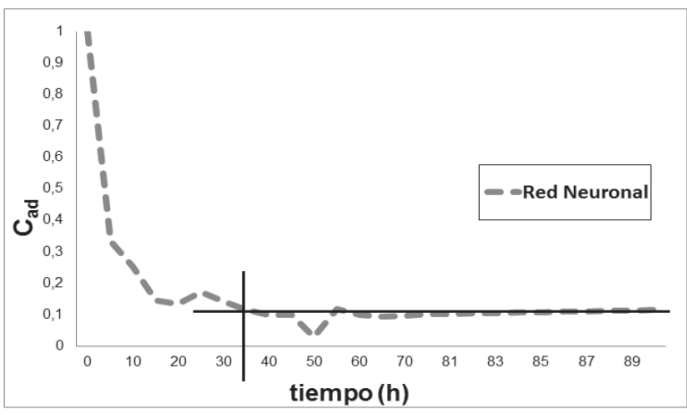

Figura 4. Ejemplo de simulación para determinación del tiempo de proceso de biodegradación teniendo en cuenta una temperatura de $30{ }^{\circ} \mathrm{C}$ y $\mathrm{pH} 5$.

En el caso de la simulación ilustrada en la Figura 4, se puede determinar, por ejemplo, que el momento perfecto del proceso fue el de $35 \mathrm{~h}$. Esto demuestra que este proceso es más eficiente que el tratamiento microbiológico, ya que de acuerdo con [22] estos procesos llevan semanas para degradar las proteínas de todos los efluentes.

Desafortunadamente, como no se encontraron otros estudios en la literatura con el mismo objetivo que el de esta investigación, no ha sido posible comparar los resultados aquí obtenidos.

\section{CONCLUSIONES}

Las papaínas fueron rápidas y eficientes en la biodegradación de proteínas de los efluentes de mataderos, en el cual se redujeron de $82 \%$ a $91 \%$ la concentración entre 30 y $40 \mathrm{~h}$ de proceso. Las redes neuronales artificiales son buenas herramientas para simular y determinar la mejor condición del proceso de biodegradación de las proteínas de efluente de matadero. Las condiciones perfectas fueron obtenidas a $\mathrm{pH} 5$, con $30^{\circ} \mathrm{C}$ y $35 \mathrm{~h}$ de proceso, en 
el cual se ha alcanzado una reducción de $91 \%$ de la concentración de proteínas.

\section{AGRADECIMIENTOS}

Agradecemos a la Universidade Nove de Julho, al PIBIQ, al CNPq y a la Universidade Federal de Sergipe, por el apoyo económico.

\section{REFERENCIAS}

[1] I.D. Coria y M. Vegas. "Determinación de la efectividad de la remediación de suelos accidentalmente contaminados con ácido sulfúrico mediante métodos geofísicos". Información Tecnológica. Vol. $22 \mathrm{~N}^{\mathrm{o}}$ 1, pp. 3-8. 2011. ISSN: 0718-0764. DOI: $10.4067 /$ S0718-07642011000100002.

[2] K.C. Passarini, F.M.C. Gamarra, R.M. Vanalle y J.C.C. Santana. "Reutilización de las aguas residuales en la irrigación de plantas y en la recuperación de los suelos". Información Tecnológica. Vol. $23 \mathrm{~N}^{\mathrm{o}} 1$, pp. 57-64. 2012. ISSN: 0718-0764. DOI: 10.4067/S0718-07642012000100007.

[3] M.E. Rodríguez, R.C. Miranda, R. Olivas y C.A. Sosa. "Efectos de las condiciones de operación sobre la biosorción de $\mathrm{Pb} 2+, \mathrm{Cd} 2+$ y $\mathrm{Cr} 3+$ en solución por Saccharomyces cerevisiae residual". Información Tecnológica. Vol. 19 $\mathrm{N}^{\circ}$ 6, pp. 47-55. 2008. ISSN: 0718-0764. DOI: $10.4067 /$ S0718-07642008000600006.

[4] P.F. Almeida, M.G.O. Araújo and J.C.C. Santana. "Collagen extraction from chicken feet for jelly production". Acta Scientiarum. Technology. Vol. $34 \mathrm{~N}^{\circ} 3$, pp. 345-351. 2012. ISSN: 1807-8621. DOI: $10.4025 /$ actascitechnol.v34i3.10602.

[5] P.F. Almeida, J.A. Salles, T.M.B. Farias y J.C.C. Santana. "Aprovechamiento de patas de pollos como alternativa para evitar los residuos generados en los mataderos". Información Tecnológica. Vol. $23 \mathrm{~N}^{\mathrm{o}} 4$, pp. 45-52. 2012. ISSN: 0718-0764. DOI: 10.4067/S0718-07642012000400006.

[6] J. Giraçol, K.C. Passarini, S.C. da Silva Filho, F.A. Calarge, E.B. Tambourgi and J.C.C. Santana. "Reduction in ecological cost through biofuel production from cooking oils: An ecological solution for the city of Campinas, Brazil". Journal of Cleaner Production. Vol. 19,
Issue 12, pp. 1324-1329. 2011. ISSN: 09596526. DOI 10.1016/j.jclepro.2011.02.015.

[7] A.F. Evangelista, N. Narain, R.R. Souza and J.C.C. Santana. "Optimization of processing parameters for the extraction of essential oil from orange rind". Acta Horticulturae. Vol. 864, pp. 479-484. 2010. ISSN: 0567-7572.

[8] P.F. Almeida, J.R. da Silva, S.C.S. Lannes, T.M.B. Farias and J.C.C. Santana. "Quality assurance and economical feasibility of an innovative product obtained from a byproduct of the meat industry in Brazil". African Journal of Business Management. Vol. 7, Issue 27, pp. 2745-2756. 2013. ISSN: 19938233. DOI: 10.5897/ AJBM2013.6977.

[9] P.F. Almeida, W.A.L. Alves, T.M.B. Farias y J.C.C. Santana. "Elaboración y clasificación sensorial de gelatinas de patas de pollos: Correlación usando redes neuronales artificiales". Información Tecnológica. Vol. 23 $\mathrm{N}^{\circ}$ 6, pp. 129-136. 2012. ISSN: 0718-0764. DOI: 10.4067/S0718-07642012000600014.

[10] M. Lado and M. Bem-Hur. "Treated domestic sewage irrigation effects on soil hydraulic properties in arid and semiarid zones: A review". Soil and Tillage Research. Vol. 106, Issue 1, pp. 152-163. 2009. ISSN: 0167-1987. DOI: $10.1016 /$ j.still.2009.04.011.

[11] A.M. Palesea, V. Pasqualeb, G. Celanoa, G. Figliuoloc, S. Masid and C. Xiloyannisa. "Irrigation of olive groves in Southern Italy with treated municipal wastewater: effects on microbiological quality of soil and fruits". Agriculture, Ecosystems \& Environment. Vol. 129, Issue 1-3, pp. 43-51. 2009. ISSN: 0167-8809. DOI: 10.1016/ j.agee.2008.07.003.

[12] A. Mazen, F.A. Faheed and A.F. Ahmed. "Study of potential impacts of using sewage sludge in the amendment of desert reclaimed soil on wheat and jews mallow plants". Brazilian Archives of Biology and Technology. Vol. 53, Issue 4, pp. 917-930. 2010. ISSN: 1516-8913. DOI: 10.1590/ S1516-89132010000400022.

[13] M. Qadir, D. Wichelns, L. Raschid-Sally, P.G. McCornick, P. Drechsel, A. Bahri and P.S. Minhas. "The challenges of wastewater irrigation in developing countries". Agricultural Water Management. Vol. 97, Issue 4, pp. 561-568. 2010. ISSN: 03783774. DOI: 10.1016/ j.agwat.2008.11.004. 
[14] K.C. Passarini, M.A. Pereira, T. M.B. Farias, F. A. Calarge and J.C.C. Santana. "Assessment of the viability and sustainability of an integrated waste management system for the city of Campinas (Brazil), by means of Ecological Cost Accounting". Journal of Cleaner Production. Vol. 65, Issue 15, pp. 479-488. 2014. ISSN: 0959-6526. DOI: 10.1016/j.jclepro.2013.08.037.

[15] R.D. Rufino, J.M. Luna, P.H.C. Marinho, C.B.B. Farias, S.R.M. Ferreira and L.A. Sarubbo. "Removal of petroleum derivative adsorbed to soil by biosurfactant rufisan produced by Candida lipolytica". Journal of Petroleum Science and Engineering. Vol. 1099, pp. 117-112. 2013. ISSN: 0920-4105. DOI: 10.1016/j.petrol.2013.08.014.

[16] M.L. Alves, B.B. Ferreira y F.R. Leta, "Evaluación de parámetros de rugosidad usando análisis de imágenes de diferentes microscopios ópticos y electrónicos". Información Tecnológica. Vol. $22 \mathrm{~N}^{\mathrm{o}} 4$, pp. 129-146. 2011. ISSN: 0718-0764. DOI: 10.4067/S0718-076420110004000014.

[17] M.R Henríquez y P.A. Palma. "Control automático de condiciones ambientales en domótica usando redes neuronales artificiales". Información Tecnológica. Vol. $22 \mathrm{~N}^{\mathrm{o}} 3$, pp. 125-139. 2011. ISSN: 0718-0764. DOI: 10.4067/S0718-07642011000300014.

[18] J.C.C. Santana, C.G. Dias, R.R. de Souza y E.B. Tamborgui. "Applying of neural network on the wine sensorial analysis from Barbados cherry". Journal of Food Process Engineering. Vol. 33, Issue 1, pp. 365-378. 2010. ISSN: 1745-4530. DOI: $10.1111 / \mathrm{j} .1745-4530.2009 .00521 . x$.

[19] D. Sendrescu and E. Bunciu. "Adaptive control of a biomethanation process using neural networks". Proceedings of the Fourth Conference on Adaptive and Self-Adaptive Systems and Applications, pp. 75-79. Nice, France. 2012. ISBN: 978-1-61208-219-6.

[20] O.B. Ayodele, H.S. Auta and N.M. Nor. "Artificial neural networks, optimization and kinetic modeling of amoxicillin degradation in photo-fenton process using aluminum pillared montmorillonite-supported ferrioxalate catalyst". Industrial \& Engineering Chemical Research. Vol. 51, Issue 50, pp. 16311-16319. 2012. ISSN: 1520-5045. DOI: 10.1021/ ie302390b.
[21] Y. Abdollahi, A. Zakaria, M. Abbasiyannejad, H.R. Masoumi, M.G. Moghaddam, K.A. Matori, H. Jahangirian and A. Keshavarzi. "Artificial neural network modeling of p-cresol photodegradation". Chemistry Central Journal. Vol. 7, Issue 1, pp. 1-7. 2013. ISSN: 1752153X. DOI: 10.1186/1752-153X-7-96.

[22] K.R.M. Prado, J.M. Rosa, J.C.C. Santana, E. Tambourgi, W.A.L. Alves and F.H. Pereira. "A bootstrapped neural network model applied to prediction of the biodegradation rate of reactive Black 5 dye". Acta Scientiarum. Technology. Vol. 35, Issue 3, pp. 565-572. 2013. ISSN: 1806-2563. DOI: $10.4025 /$ actascitechnol. v35i3.16210.

[23] J.M. Rosa, K.R.M. Prado, W.A.L. Alves, F.H. Pereira, J.C.C. Santana and E. Tambourgi. "Applying of a neural network in effluent treatment simulation as an environmental solution for textile industry". Chemical Engineering Transactions. Vol. 32, pp. 73-78. 2013. ISSN: 1974-9791. DOI: $10.3303 /$ CET1332013.

[24] R.K. Singh and P.R. Kumar. "Development of artificial neural network modeling of p-cresol biodegradation". International Journal of Advanced Biotechnology and Research. Vol. 5, Issue 1, pp. 43-53. 2014. ISSN: 2278-599X.

[25] P. Kianmehr, W. Mansoor and F.A. Kfoury. "Prediction of biogas generation profiles in wastewater treatment plants using neural networks". Vol. 2, Issue 3, pp. 201-205. 2014. ISSN: 1793-821X. DOI: 10.7763/JOCET.

[26] U.A. Lima, E. Aquarone, W. Bprzani and W. Schmidell. "Biotecnologia industrial. Processos fermentativos e enzimáticos". Edgard Blücher Ltda. São Paulo, Brasil. Vol. 3, pp. 616. 2001. ISBN: 8521202806.

[27] A. Cadogan and J. Hanks. "Microbiology and biotecnology: biology advanced studies". Thomas Nelson and Sons. Croatia, pp. 136. 1995. ISBN: 0174482272.

[28] M.M. Bradford. "A rapid and sensitive method for the quantitation of microgram quantities of proteins. Utilizing the principle of protein-dye binding". Analytical Biochemistry. Vol. 72, pp. 248-254. 1976. ISSN: 0003-2697. DOI: 10.1016/0003-2697(76)90527-3.

[29] S. Russel and P. Norvig. "Artificial intelligence a modern approach". Prentice Hall. Third edition, pp. 960. New Jersey, USA. 1995. ISBN: 0131038052. 